\title{
Music Perception of Cochlear Implant Users Compared with that of Hearing Aid Users
}

\author{
Valerie Looi, ${ }^{1,2,3}$ Hugh McDermott, ${ }^{1}$ Colette McKay, ${ }^{1,4}$ and Louise Hickson ${ }^{5}$
}

\begin{abstract}
Objective: To investigate the music perception skills of adult cochlear implant (CI) users in comparison with hearing aid (HA) users who have similar levels of hearing impairment. It was hypothesized that the HA users would perform better than the CI recipients on tests involving pitch, instrument, and melody perception, but similarly for rhythm perception.
\end{abstract}

Design: Fifteen users of the Nucleus CI system and 15 HA users participated in a series of music perception tests. All subjects were postlingually deafened adults, with the HA subjects being required to meet the current audiological criteria for CI candidacy. A music test battery was designed for the study incorporating four major tasks: (1) discrimination of $\mathbf{3 8}$ pairs of rhythms; (2) pitch ranking of one-octave, half-octave, and quarter-octave intervals; (3) instrument recognition incorporating three subtests, each with 12 different instruments or ensembles; and (4) recognition of 10 familiar melodies. Stimuli were presented via direct audio input at comfortable presentation levels. The test battery was administered to each subject on two separate occasions, approximately 4 mo apart.

Results: The results from the rhythm test were $93 \%$ correct for the CI group and 94\% correct for the HA group; these scores were not significantly different. For the pitch test, there was a significant difference between the HA group and the CI group $(p<0.001)$, with higher mean scores recorded by the HA group for all three interval sizes. The CI subject group was unable to rank pitches a quarter-octave apart, only scoring at chance level for this interval size. In the instrument recognition test, although there was no significant difference between the mean scores of the two groups, both groups obtained significantly higher scores for the subtest incorporating single instrument stimuli than those incorporating multiple instrumentations $(p<0.001)$. In the melody test, there was a significant difference between the implantees' mean score of $52 \%$ correct and the HA group's mean of $91 \%(p<0.001)$.

Conclusions: As hypothesized, results from the two groups were almost identical for the rhythm test,

\footnotetext{
${ }^{1}$ Department of Otolaryngology, The University of Melbourne, Australia; ${ }^{2}$ Cooperative Research Centre for Cochlear Implant and Hearing Aid Innovation, Australia; ${ }^{3}$ Department of Communication Disorders, The University of Canterbury, New Zealand; ${ }^{4}$ School of Health and Life Sciences, Aston University, United Kingdom; and ${ }^{5}$ Division of Audiology, School of Health and Rehabilitation Sciences, The University of Queensland, Australia.
}

with the HA group performing significantly better than the CI group on the pitch and melody tests. However, there was no difference between the groups in their ability to identify musical instruments or ensembles. The results of this study indicate that HA users with similar levels of hearing loss perform at least equal to, if not better than, CI users on these music perception tests. However, despite the differences between scores obtained by the CI and HA subject groups, both these subject groups were largely unable to achieve accurate or effective music perception, regardless of the device they used.

(Ear \& Hearing 2008;29;1-•)

\section{INTRODUCTION}

It is well established that people with hearing impairment, including cochlear implant (CI) and hearing aid (HA) users, perceive musical rhythm approximately as well as those with normal hearing (NH) (Darrow, 1979, 1984; Gfeller \& Lansing, 1991, 1992; Gfeller, et al., 1997; Klajman, et al., 1982; Korduba, 1975; Schulz \& Kerber, 1994). However, the performance of CI users on other music perception tasks such as instrument identification, melody recognition, and pitch discrimination is far less adequate (Dorman, et al., 1991; Fujita \& Ito, 1999; Gfeller \& Lansing, 1991, 1992; Gfeller, et al., 1998, 2002a,c; Leal, et al., 2003; Schulz \& Kerber, 1994). In contrast, there are very few studies of the perception of music through HAs for adults with severe to profound hearing losses. The present study aimed to investigate the music perception skills of adult CI users compared with adult HA users who met the audiological criteria for cochlear implantation. This was achieved through a series of music tests assessing rhythm, pitch, timbre, and melody perception.

A study was published by Kong et al. (2005) in which melody recognition was assessed with five CI users who had residual hearing in the nonimplanted ear (i.e., they used an HA in the contralateral ear). After a practice session, the subjects were tested in three listening conditions (CI-alone, HA-alone, and CI with HA), on three sets of 12 familiar melodies devoid of rhythm cues, encompassing three different frequency ranges (low, mid, and high). Closed-set recognition of the loudspeaker-presented stimuli was evaluated, with subjects receiving immediate visual feedback as to the accuracy of their responses. Re- 
sults were highly variable, both within and between subjects, ranging from $19 \%$ to $90 \%$ correct for the HA-alone condition, $8 \%$ to $81 \%$ correct for the CIalone condition, and $21 \%$ to $92 \%$ for the combined modalities. The mean HA-alone score was significantly better than the average CI-alone score, with little difference between performance in the HA-alone condition and the HA with CI condition. That is, the HA provided better melody recognition scores than the CI in that study. Whereas the subjects in Kong et al. study were CI users who used an HA in their contralateral ear, the current study recruited two separate subject groups to compare the two devices across a wider range of musical tasks. The CI subject group was tested while listening with only their CI, and the HA subject group did not have a CI.

In a study including only CI users, Gfeller \& Lansing (1992) used the Primary Measures of Music Audiation (PMMA) to assess tonal and rhythm perception in 34 subjects. The PMMA, a standardized test of musical perception for children developed by Gordon (1986), comprises two subtests (tonal and rhythm), each incorporating 40 stimuli pairs to be assessed as "same" or "different" by the listener. In Gfeller \& Lansing (1992) study, the CI subjects scored 78\% correct on the tonal subtest and $85 \%$ for the rhythm subtest, and they reported the tonal subtest to be more difficult. Fujita \& Ito (1999) found that while five of eight postlingually deaf adult CI users accurately discriminated the higher of two notes between 4 and 10 semitones apart, the other three subjects could not discriminate between two notes one octave (12 semitones) apart. It should be noted, however, that both these studies primarily used older, nowobsolete, and speech-processing strategies.

Leal et al. (2003) conducted two pitch perception tasks with 29 Nucleus CI24 users, 20 using the ACE strategy and 9 using the SPEAK strategy. The first task was a same/different comparison of 12 pitch pairs. The second task consisted of eight paired musical excerpts where the listener was asked to state whether the pitch became higher or lower, and where this change occurred. For the same/different comparison, the mean score was $90 \%$ correct, with $69 \%$ of subjects scoring above $88 \%$. In the higher/ lower task, subjects averaged $74 \%$ correct, with around half of the subjects scoring above $75 \%$.

Researchers have shown that CI users perform significantly more poorly, on average, than NH listeners at perceiving timbre (Gfeller, et al., 1998, 2002c; Schulz \& Kerber, 1994). Timbre perception is usually assessed via instrument identification tests. Gfeller et al. (1998) found that NH subjects were not only significantly more accurate than implantees in recognizing each of four different musical instruments, but they also seemed to be more confident with their responses. In a larger instrument recognition study, Gfeller et al. (2002c) found a significant difference between $\mathrm{CI}$ and $\mathrm{NH}$ subjects' ability to recognize eight different musical instruments playing the same seven-note melodic sequence with equal-duration notes. Subjects were also asked to appraise the overall pleasantness of the eight instruments using a visual analog scale. $\mathrm{NH}$ subjects scored $91 \%$ correct on the recognition task, whereas the CI patients scored only $47 \%$ correct, with correspondingly lower general appraisal scores (Gfeller et al., 1998). Schulz \& Kerber study using single-channel CIs in conjunction with a now-obsolete analog sound-processing scheme also found that $\mathrm{NH}$ subjects scored significantly more accurately than CI subjects (90\% and $36 \%$, respectively) in a five-instrument closed-set recognition test.

Another major component incorporated into many music perception test batteries is the recognition of melodies. Although pitch perception is an integral part of melody recognition, it is by no means the only element to consider; for example, the perception of rhythm, lyrics, timbre, genre, or musical style must also be considered. Schulz \& Kerber (1994) reported that CI subjects found rhythmically structured tunes easier to recognize than nonrhythmic tunes, whereas Fujita \& Ito (1999) reported that the inclusion of vocal cues improved melody recognition scores. In Leal et al. (2003) study, only one of the 29 subjects scored above $50 \%$ correct in a closed-set recognition task comprising eight familiar melodies played by an orchestra. When the test was simplified to a solo piano presentation, the number of subjects scoring at least $50 \%$ correct increased to 14 , further improving to 28 of the 29 subjects with the addition of verbal cues. In a larger study, Gfeller et al. (2002a) presented 12 familiar melodies along with 12 foils (i.e., modified versions of the original melody) to $\mathrm{NH}$ and CI subjects. They found that $\mathrm{NH}$ subjects identified significantly more melodies than CI subjects (mean scores of $55 \%$ and $13 \%$ correct, respectively). Two-thirds of the melodies correctly identified by CI subjects were classified by the researchers to be highly rhythmic in nature. The authors surmised that melody recognition involves a multitude of perceptual and cognitive skills, including pitch and rhythm perception.

Although several authors have speculated that HA users may perform differently, on average, from CI users, no definitive research has verified this. The main hypothesis of the present study was that HA users who met the CI candidacy criteria would perform better than CI recipients on pitch discrimination, instrument identification, and melody recognition tests, but similarly on rhythm perception tests. 


\section{Methods}

\section{Subjects}

Although the aim of this research was to compare the performance of CI users with HA users on a variety of music perception tasks, it was important to verify the music tests initially with a group of listeners with $\mathrm{NH}$, to ensure that the tests were appropriate for administration with the subjects with hearing impairments. Therefore, 10 adults with NH (7 females, 3 males) were recruited. They ranged from 22 to 51 yrs (mean, $32.9 \mathrm{yrs}$ ), and had bilateral hearing thresholds $25 \mathrm{~dB}$ HL or better at octave intervals between 250 and $8000 \mathrm{~Hz}$.

The CI subject group consisted of 15 postlingually deafened adult users of the Nucleus CI system (8 CI24, 7 CI22), ranging from 36 to 75 yrs (mean, 60.4 yrs). There were eight subjects using the ACE strategy, and seven using the SPEAK strategy. Further details of the CI subject group are given in Table 1 .

The HA subject group comprised 15 postlingually deafened adult HA users, ranging from 49 to $80 \mathrm{yrs}$ (mean, $64.7 \mathrm{yrs}$ ). HA subjects used their own aids, and were selected to meet the same CI criteria as the subjects in the CI group, in terms of level of hearing loss and speech perception scores. For the Australian clinics involved in this study, this criteria were having a bilateral moderately severe to profound sensorineural hearing loss between 1 and $4 \mathrm{kHz}$ (i.e., hearing thresholds worse than $55 \mathrm{~dB} \mathrm{HL}$ ), and speech perception scores for sentence stimuli presented auditory-alone in quiet at $65 \mathrm{~dB}$ SPL gener- ally $\leq 70 \%$ in the best-aided condition, and $\leq 40 \%$ in the ear to be implanted. Details of the HA subject group are given in Table 2.

\section{Music Test Battery}

A music test battery was designed by the researchers for the purpose of this study. Three fundamental components of music perception (rhythm, pitch, and instrument identification) were assessed, along with a melody recognition test where both pitch and rhythm cues were preserved. For all the tests outlined below, subjects selected their preferred listening settings on their device, and presentation levels were individually verified to be of a comfortable loudness.

Rhythm test - The stimuli for this test were derived from Gordon (1986) PMMA rhythm subtest. Because of the difficulties identified in Gfeller \& Lansing (1992) study, the verbal prompts present in the original recording were eliminated for this study. The test consisted of 38 pairs of short rhythm excerpts created using tones of the same pitch. In each pair, the excerpts were separated by $1.5 \mathrm{sec}$ of silence. Subjects were required to assess whether each pair of rhythms was the same or different, with a score out of 38 being obtained.

Pitch test $\bullet$ This test comprised three subtests, each identical in format but using differing interval sizes. The first subtest consisted of note pairs one octave (12 semitones) apart, the second subtest comprised half-octave (6 semitones) intervals, and the third subtest comprised quarter-octave (3 semi-

TABLE 1. Cochlear implant subject details

\begin{tabular}{|c|c|c|c|c|c|c|c|c|c|c|c|}
\hline $\begin{array}{l}\text { Subject } \\
\text { (Sex) }\end{array}$ & $\begin{array}{l}\text { Age } \\
\text { (yrs) }\end{array}$ & Aetiology & $\begin{array}{c}\text { Duration } \\
\text { profound } \\
\text { hearing } \\
\text { loss (yrs) }\end{array}$ & $\begin{array}{c}\text { Device } \\
\text { experience } \\
\text { (mos) }\end{array}$ & $\begin{array}{c}\text { Speech } \\
\text { perception } \\
\text { score }(\%)^{*}\end{array}$ & $\begin{array}{c}\text { Music } \\
\text { experience } \\
\text { score† }\end{array}$ & $\begin{array}{c}\text { Ear } \\
\text { implanted }\end{array}$ & $\begin{array}{c}\text { Type of } \\
\text { Cl }\end{array}$ & Processor & Strategy & $\begin{array}{c}\text { HA worn in } \\
\text { contralateral } \\
\text { ear }\end{array}$ \\
\hline $1(\mathrm{M})$ & 47 & $\mathrm{C} / \mathrm{P}$ & 2 & 16 & 95 & 2 & $\mathrm{R}$ & $24 \mathrm{R}$ & Sprint & Ace $1200 \mathrm{~Hz}$ & No \\
\hline $2(\mathrm{M})$ & 67 & Otosclerosis & 10 & 60 & 75 & 2 & $\mathrm{~L}$ & $24 \mathrm{M}$ & Sprint & Ace $1200 \mathrm{~Hz}$ & No \\
\hline $3(F)$ & 45 & $\mathrm{C} / \mathrm{P}$ & 5 & 22 & 99 & 2 & $\mathrm{R}$ & $24 \mathrm{M}$ & Sprint & Ace $1800 \mathrm{~Hz}$ & No \\
\hline $4(F)$ & 36 & Rubella & 10 & 108 & 61 & 0 & $\mathrm{R}$ & $22 \mathrm{M}$ & Esprit22 & Speak & No \\
\hline $5(F)$ & 72 & $\mathrm{C} / \mathrm{P}$ & 14 & 24 & 96 & 1 & $\mathrm{~L}$ & $24 \mathrm{M}$ & Esprit3G & Ace $720 \mathrm{~Hz}$ & Yes \\
\hline $6(F)$ & 56 & $\mathrm{C} / \mathrm{P}$ & 11 & 17 & 100 & 1 & $\mathrm{~L}$ & $24 \mathrm{R}$ & Esprit3G & Ace $900 \mathrm{~Hz}$ & Yes \\
\hline $7(\mathrm{M})$ & 71 & Trauma & 27 & $300 \S$ & 97 & 2 & $L$ & $24 \mathrm{M}$ & Sprint & Ace $275 \mathrm{~Hz}$ & No \\
\hline $8(F)$ & 75 & $\mathrm{C} / \mathrm{P}$ & 11 & 38 & 95 & 2 & $\mathrm{R}$ & $24 \mathrm{R}$ & Esprit3G & Ace $900 \mathrm{~Hz}$ & Yes \\
\hline $9(F)$ & 70 & $\mathrm{C} / \mathrm{P}$ & 9 & $180 \S$ & 84 & 0 & $\mathrm{~L}$ & $22 \mathrm{M}$ & Esprit22 & Speak & No \\
\hline $10(F)$ & 61 & $\mathrm{C} / \mathrm{P}$ & 7 & 18 & 78 & 0 & $L$ & $24 \mathrm{R}$ & Esprit3G & Ace $500 \mathrm{~Hz}$ & Yes \\
\hline $11(\mathrm{M})$ & 48 & $\mathrm{C} / \mathrm{P}$ & 14 & 135 & 72 & 0 & $\mathrm{R}$ & $22 \mathrm{M}$ & Esprit22 & Speak & No \\
\hline $12(F)$ & 66 & Meningitis & 18 & 211 & 90 & 2 & $\mathrm{R}$ & $22 \mathrm{M}$ & Esprit3G & Speak & No \\
\hline $13(\mathrm{M})$ & 69 & $\mathrm{C} / \mathrm{P}$ & 12 & 138 & 37 & 0 & $L$ & $22 \mathrm{M}$ & Esprit22 & Speak & No \\
\hline $14(\mathrm{M})$ & 64 & $\mathrm{C} / \mathrm{P}$ & 30 & 184 & 79 & 0 & $\mathrm{~L}$ & $22 \mathrm{M}$ & Spectra & Speak & No \\
\hline $15(\mathrm{M})$ & 59 & Trauma & 32 & 185 & 94 & 0 & $\mathrm{~L}$ & $22 \mathrm{M}$ & Spectra & Speak & No \\
\hline Mean & 60.4 & & 14.1 & 109.1 & 83.9 & 0.93 & & & & & \\
\hline
\end{tabular}

* Word score (\%) on CUNY sentence test, tested auditory alone, binaurally at $65 \mathrm{~dB}$ SPL.

$\dagger$ Levels of music participation, categorized from 0 (no experience) to 2 (most experienced). Ascertained via a questionnaire developed for this research

$\ddagger 24 R$ = Nucleus Cl24, Contour electrode array; $24 M=$ Nucleus Cl24, straight electrode array; 22M = Nucleus Cl22.

$\S$ Subjects had been reimplanted; total number of months with both devices report.

C/P, congenital/progressive. 
TABLE 2. Hearing aid subject details

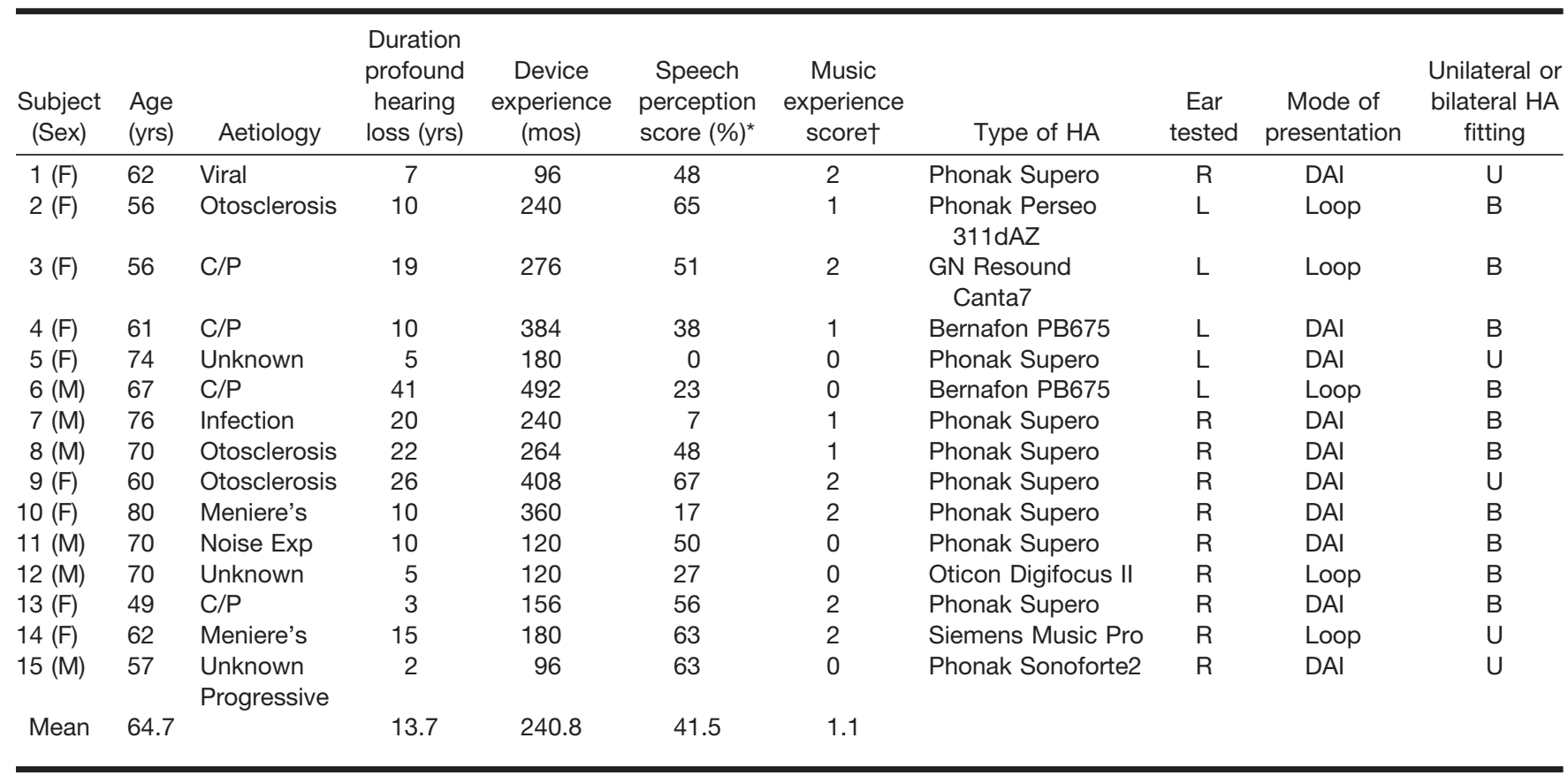

* Word score (\%) on CUNY sentence test, best aided condition (i.e., binaural or better-hearing ear), auditory alone at $65 \mathrm{~dB} S \mathrm{SPL}$.

t Levels of music participation, categorized from 0 (no experience) to 2 (most experienced). Ascertained via a questionnaire developed for this research

$C / P$, congenital/progressive; DAI, direct audio input; Loop, neck loop system in conjunction with the telecoil; $U$, unilateral; $B$, bilateral.

tones) intervals. Recordings of the vowels /i/ and /a/ were obtained from trained male and female singers with the two notes comprising each stimulus pair being of the same vowel, and sung by the same singer at the designated interval size. Table 3 summarizes the fundamental frequencies of the stimuli. For half of the presentations, the first note was higher than the second (descending), with the other half being ascending. Subjects were required to select the higher note for each stimulus pair. The one-octave and half-octave subtests provided scores out of 96, whereas the quarter-octave subtest was scored out of 128 . To reduce the potential for loudness cues to influence perceptual judgments, the levels of the individual notes constituting each pair were presented randomly at either the subject-determined comfortable loudness level, or $6 \mathrm{~dB}$ below this.
Instrument test - This test consisted of three subtests, each with the same procedure but different stimuli. The first subtest consisted of single instrument sounds, the second comprised solo instruments with background accompaniment, and the third involved music ensembles. For each subtest, four 5-sec extracts of 12 different instruments or ensembles were included (i.e., 48 stimuli per test). Each of these extracts were different (i.e., from a different musical piece, or a different section of the same piece), and were obtained from high quality, commercially available compact disc sound recordings of music works. To minimize any unwanted effects of loudness cues, the levels of the four extracts of each instrument or ensemble were randomized over a $6 \mathrm{~dB}$ range below the subject-determined comfortable loudness level.

TABLE 3. Fundamental frequencies of stimuli tested in the pitch test

\begin{tabular}{|c|c|}
\hline Interval size & Fundamental frequency of stimuli used for each interval \\
\hline Female & C4-C5 (262-523 Hz); D4-D5 (311-622 Hz); F4-F5 (370-740 Hz) \\
\hline \multicolumn{2}{|c|}{ Subtest 2 , half octave } \\
\hline Female & C4-F4 (262-370 Hz); F4-C5 (370-523 Hz); C5-F5 (523-740 Hz) \\
\hline Male & G2-C3 (98-139 Hz); C3-G3 (139-196 Hz); G3-C4 (196-277 Hz) \\
\hline Male & C3-E3 (139-165 Hz); E3-G3 (165-196 Hz); G3-A3 (196-233 Hz); A3-C4 (233-277 Hz) \\
\hline
\end{tabular}


In subtest 1, 12 commonly heard individual instruments were selected covering the four instrumental families (strings, woodwind, brass, and percussion) and a wide pitch range. The instruments were male singer, female singer, piano, guitar, bass drum (or timpani), drum kit, xylophone, cello, violin, trumpet, flute, and clarinet. The drum kit stimuli incorporated the use of hi-hats, cymbals, and tomtoms, as opposed to just the lower-frequency "thuds" of the bass drum stimuli. In subtest 2, the 12 instruments from subtest 1 were presented to subjects in a different context. This subtest used the instruments in a solo role, with background musical accompaniment (i.e., an orchestra). In subtest 3, 12 different music ensembles, each playing as a cohesive, unified group, were included: choir (four-part, a capella), orchestra, jazz band (instrumental only), rock band (instrumental only), country and western band (instrumental only), string quartet, percussion ensemble (varying instrumental combinations), violin and piano duet, cello and piano duet, male singer and piano duet, female singer and piano duet, and one male and one female singer with piano accompaniment.

For each subtest, subjects were presented with a list of the 12 instruments or ensembles, each with a matching picture. They were instructed to name the instrument or ensemble that they thought was playing. As each instrument or ensemble was presented four times, a score out of 48 was obtained for each subtest. For the second subtest (i.e., solo instrument with background accompaniment), two runs were conducted to assess whether the supplementary information pertaining to the background accompaniment affected music perception. In the first run, subjects were asked to identify the solo instrument from the same list as used in subtest 1 . For the second run, subjects were additionally informed that the background ensemble was an orchestra, before being required to identify the instrument from the same closed-set list. This allowed the effect of prior knowledge to be assessed. The same stimuli were used for both runs.

Melody test $\bullet$ The first 15 sec of 10 well-known melodies were recorded on a Yamaha PSR-276 portable keyboard, with the original rhythms being preserved. These melodies were chosen as they had been identified in a previous study as 10 of the most familiar melodies to an Australian population (Looi, et al., 2003). The melodies were as follows: Advance Australia Fair, Baa Baa Black Sheep, For He's a Jolly Good Fellow, Happy Birthday, Jingle Bells, O Come All Ye Faithful, Old Macdonald Had a Farm, Silent Night, Twinkle Twinkle Little Star, and Waltzing Matilda. Each melody was played in C major (centering around $\mathrm{C} 4$, middle-C) at an appropriate speed. All notes for each melody fell in the range from C3 to C5 $(131-523 \mathrm{~Hz})$, and where the song consisted of both a verse and chorus, only the chorus was recorded. The last $5 \mathrm{sec}$ of each melody was linearly ramped to zero amplitude. After each melody had been presented, subjects were asked to name the melody they thought was playing from a list of the 10 melody titles. Each melody was presented twice, and a score out of 20 was obtained.

\section{Overall Procedure}

Before testing the CI and HA subjects, the validity of the test battery was checked with the group of 10 adults with $\mathrm{NH}$, who undertook the quarteroctave pitch test, the three closed-set instrument identification tests, and the melody recognition test. The one-octave and half-octave pitch tests were not tested for time-efficiency. As it was expected that adults with $\mathrm{NH}$ would be able to reliably rank pitches a quarter-octave apart, and therefore for a "ceiling effect" to be observed in the results, it was assumed that testing of larger, and hence easier, intervals was not necessary. For the test battery verification assessments, the stimuli were presented via Etymotic ER4B earphones that have a flat frequency response.

All the subjects in both the CI and HA subject groups completed a questionnaire about previous music training and participation levels, with the responses being used to determine a music experience score as reported in Tables 1 and 2. Subjects were ranked into three categories: "0" indicates having had no formal music training or participation in music activities or music classes; " 1 " indicates having had instrumental lessons for 2 yrs or less, and/or participation in music activities or music classes for 5 yrs or less; and "2" indicates having had formal instrumental lessons for more than $2 \mathrm{yrs}$, and/or participation in informal music activities or music classes for more than 5 yrs.

The entire test battery took approximately $3 \mathrm{hrs}$ to complete, and was conducted over two to three sessions with each subject. The entire test battery was administered to the subjects in the CI and HA groups on two occasions approximately 4 mo apart. The order of presentation of the stimuli in each individual test or subtest was randomized, with the overall order of the tests within the battery being pseudorandomized.

As testing was conducted at two different sites, the signals were not presented to subjects in the sound field to avoid the effects of variable loudspeaker frequency responses and room acoustics. Sound-treated rooms or sound-isolation booths were used for all of the testing. All stimuli were presented from a Dell "Latitude" laptop computer, connected to 
an external Creative "Soundblaster Extigy" soundbox. For the CI subjects, stimuli were delivered to their speech processors via direct audio input. For the HA subjects, direct audio input via an audio shoe was used where available, although a neckloop system operating via the telecoil on the HA was needed in some instances (Table 2). For the HA subjects, tests were presented to the ear that had better speech perception scores, or in cases with similar or fluctuating losses, to the ear that the subject preferred. Stimuli were not repeated and no feedback was provided. Subject's responses were entered directly into the computer for postsession analysis. To ensure that it was identification abilities being assessed, and not musical knowledge for the instrument perception and melody recognition tests, each subject's familiarity with the instruments, ensembles, and melodies was verified before testing. Subjects were verbally asked to indicate which instrument, ensemble, or melody they had not heard before implantation, when they had better hearing. All the subjects were familiar with all the instruments and melodies used in the test battery.

\section{Results}

The mean scores for the group of adults with $\mathrm{NH}$ who verified the music test battery were greater than $95 \%$ correct for each of the tests or subtests assessed. The CI and HA group's scores for each of the test bocks, along with the combined test block scores are presented in Tables 4 and 5, respectively. Comparisons between the scores from the two test

TABLE 4. Results from the $\mathrm{Cl}$ subject group (\% correct)

\begin{tabular}{|c|c|c|c|c|c|c|}
\hline & \multicolumn{2}{|c|}{ Test block 1} & \multicolumn{2}{|c|}{ Test block 2} & \multicolumn{2}{|c|}{$\begin{array}{l}\text { Combined } \\
\text { test blocks }\end{array}$} \\
\hline & Mean & SD & Mean & SD & Mean & SD \\
\hline Rhythm & 92.8 & 3.1 & 92.8 & 3.7 & 92.8 & 3.3 \\
\hline \multicolumn{7}{|l|}{ Pitch } \\
\hline One-octave* & 69.4 & 16.6 & 68.2 & 17.6 & 68.0 & 16.8 \\
\hline Half-octave & 62.5 & 8.2 & 66.0 & 9.7 & 64.3 & 9.0 \\
\hline Quarter-octave & 51.1 & 6.3 & 52.4 & 7.4 & 51.8 & 6.8 \\
\hline $\begin{array}{l}\text { Instrument } \\
\text { Single instrument }\end{array}$ & 58.9 & 11.9 & 62.8 & 11.5 & 60.8 & 11.7 \\
\hline $\begin{array}{l}\text { Instrument with } \\
\text { background }\end{array}$ & 43.9 & 11.9 & 46.8 & 13.3 & 45.3 & 12.6 \\
\hline Ensemble & 41.3 & 14.6 & 43.9 & 15.1 & 42.6 & 14.7 \\
\hline Melody & 49.0 & 23.5 & 54.7 & 24.9 & 51.8 & 23.9 \\
\hline
\end{tabular}

The means (\% correct scores), and SD (percentage-points) for both test blocks individually, as well as the average when the scores for each subject from the two test blocks were combined are provided. The "Instrument with Background" subtest score is the average of the scores obtained from the two runs conducted for each test block.

${ }^{*}$ For the one-octave pitch subtest, only $12 \mathrm{Cl}$ subjects undertook test block 1 , whereas all $15 \mathrm{Cl}$ subjects undertook test block 2. Three of the Cl subjects were not available to undertake this subtest during the first test block. For the results in this table, the test block 1 results for the one-octave subtest were for 12 subjects, with the test block 2 and combined test blocks results being the scores across all 15 subjects.
TABLE 5. Results from the HA subject group (\% correct)

\begin{tabular}{|c|c|c|c|c|c|c|}
\hline & \multicolumn{2}{|c|}{ Test block 1} & \multicolumn{2}{|c|}{ Test block 2} & \multicolumn{2}{|c|}{$\begin{array}{l}\text { Combined } \\
\text { test blocks }\end{array}$} \\
\hline & Mean & SD & Mean & SD & Mean & SD \\
\hline Rhythm & 94.2 & 4.8 & 94.0 & 3.7 & 94.1 & 4.2 \\
\hline \multicolumn{7}{|l|}{ Pitch } \\
\hline One-octave & 86.6 & 11.8 & 93.5 & 6.2 & 90.2 & 9.9 \\
\hline Half-octave & 80.2 & 10.4 & 87.2 & 7.0 & 83.7 & 9.4 \\
\hline Quarter-octave & 71.7 & 12.2 & 77.8 & 10.1 & 74.7 & 11.5 \\
\hline \multicolumn{7}{|l|}{ Instrument } \\
\hline Single instrument & 65.0 & 14.7 & 72.1 & 12.1 & 68.5 & 13.7 \\
\hline $\begin{array}{l}\text { Instrument with } \\
\text { background }\end{array}$ & 49.8 & 15.6 & 53.8 & 17.7 & 51.8 & 16.7 \\
\hline Ensemble & 44.7 & 19.5 & 48.8 & 19.8 & 46.7 & 19.4 \\
\hline Melody & 89.3 & 16.4 & 91.7 & 16.1 & 90.5 & 16.0 \\
\hline
\end{tabular}

The means (\% correct scores), and SD (percentage-points) for both test blocks individually, as well as the average when the scores for each subject from the two test blocks were combined are provided. The "Instrument with Background" subtest score is the average of the scores obtained from the two runs conducted for each test block.

blocks for the CI and HA groups, and the potential of a learning effect impacting on the results, will be addressed later in this section.

\section{Subjects Compared With HA Subjects}

The CI and HA subject groups' mean scores across the two test runs for the music test battery are shown in Figure 1. To simplify the between-group comparisons, for these scores and the analyses in this section, the scores from each subject's two runs of the test battery were averaged. Two-tailed statistical tests were applied to compare the two subject groups.

\section{Subject Factors}

Independent-samples $t$ tests showed that there were no significant differences between the CI and HA groups for the subject factors of age or music experience levels. There were significant differences

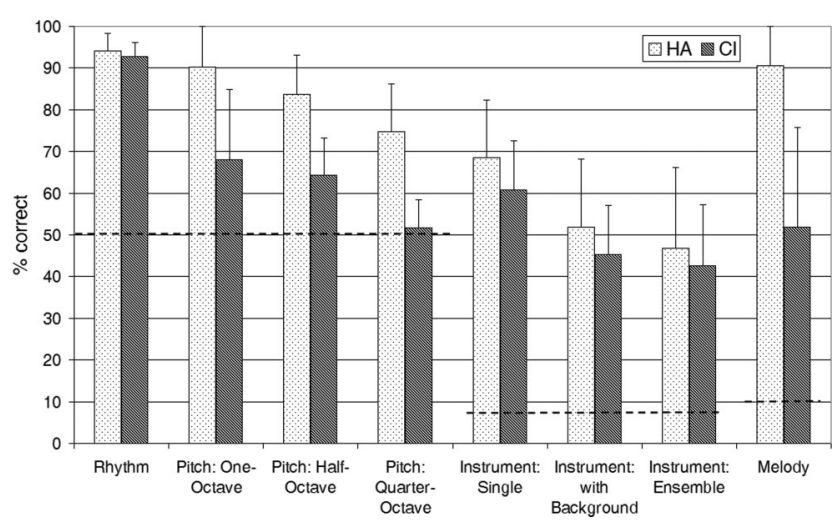

Fig. 1. Music test scores comparison. Error bars indicate one standard deviation. The dashed line indicates the chance scores for the different tests. 
between the groups for the factors of experience with the device, and aided speech perception scores, though, with the CI subjects obtaining significantly higher speech perception scores, despite having less experience with the device. Considering that the HA subjects were required to meet the audiological criteria for a CI, the difference between speech perception scores is largely expected.

\section{Rhythm Test}

The CI group averaged $93 \%$ correct $(\mathrm{SD}=2.53$ percentage-points) on this test, with the HA group averaging $94 \%$ correct (SD $=3.58$ percentagepoints). An independent-samples $t$ test showed no significant difference between the groups' scores $(p=0.255)$.

\section{Pitch Test}

The results from this test are summarized in Table 6. For all three subtests, the HA group scored significantly higher than the CI group when comparing the average percent-correct score across the four vowels (i.e., the total score). Large SDs were observed for both subject groups in all three subtests, along with differences between scores obtained for female-sung compared with male-sung vowels.

A three-way repeated-measures analysis of variance (ANOVA) was conducted using the betweensubject factor of group (i.e., CI versus HA subjects), within-subject factors of interval size (i.e., one-octave, half-octave, and quarter-octave), and singer's sex (i.e., male-sung versus female-sung vowels). This showed highly significant main effects of group $(p<0.001)$ and interval size $(p<0.001)$. As would be expected, average scores decreased with the smaller interval sizes. Although there was no main effect of singer sex $(p=$ $0.96)$, there was a significant two-way interaction between group and singer sex $(p<0.001)$.

In view of this significant interaction, separate two-way repeated-measures ANOVAs were conducted for the CI and HA groups to compare the within-subject factors of interval size and singer's sex. This showed that the CI group performed significantly better with the male-sung than with the female-sung stimuli ( $p=0.035)$, whereas the HA group was significantly better with the female-sung stimuli ( $p=0.001)$.

One-sample $t$ tests were also calculated to compare each subject group's performance to the chance score of $50 \%$. This revealed that the CI group's average for the quarter-octave interval $(51.75 \%$ correct) was not significantly different to chance-level performance ( $p=0.238$ ). That is, as a group, the CI subjects were unable to discriminate the pitch of two notes a quarter-octave apart. Their half-octave $(64.27 \%$ correct) and one-octave $(67.98 \%$ correct $)$ subtest scores were significantly better than the chance score $(p<0.001$, and $p=0.001$, respectively). For the HA group, performance for all three interval sizes was significantly above the chancelevel score ( $p<0.001$ for all comparisons).

\section{Instrument Test}

The mean percent-correct scores for the CI group for subtests 1 (single instrument), 2 (instrument with background accompaniment), and 3 (ensembles) were $61 \%, 45 \%$, and $43 \%$, respectively, whereas for the HA group they were $69 \%, 52 \%$, and $47 \%$, respectively. To compare the groups' performances across the three subtests, a two-way repeated-measures ANOVA was conducted. This showed no significant difference for the between-subject factor of group ( $p=0.222$ ), but a significant difference for the within-subject factor of subtest $(p<0.001)$. There was no significant two-way interaction between these two terms ( $p=0.529$ ). For the effect of subtest, post hoc pairwise comparisons with Bonferroni corrections showed the differences between subtests 1 and 2 , as well as between subtests 1 and 3 to be statistically significant ( $p<0.001$ in both cases). The difference between subtests 2 and 3 was approaching significance $(p=0.062)$. That is, the HA and CI groups performed significantly better at

TABLE 6. Pitch test results

\begin{tabular}{|c|c|c|c|c|c|c|c|c|c|}
\hline & \multicolumn{3}{|c|}{ One octave subtest } & \multicolumn{3}{|c|}{ Half octave subtest } & \multicolumn{3}{|c|}{ Quarter octave subtest } \\
\hline Mean & 68.0 & 77.8 & 59.9 & 64.3 & 64.2 & 64.4 & 51.8 & 55.8 & 47.7 \\
\hline SD & 17.3 & 22.7 & 18.4 & 8.5 & 9.9 & 10.5 & 5.5 & 6.0 & 9.1 \\
\hline \multicolumn{10}{|c|}{ HA subjects } \\
\hline
\end{tabular}

Both-singers mean, male-vowel mean, and female-vowel mean \% correct scores, and standard deviations (SD) for the three pitch subtests. The male-vowel mean was the average of the la/ and lil vowels sung by the male singer for the respective subtests, and the female-vowel mean was the average of the la/ and li/ vowels sung by the female singer. 
identifying instruments in the single-instrument subtest than for the other two subtests incorporating multiple instrumentation.

\section{Melody Test}

CI subjects averaged 52\% correct (SD: 23.59 percentage-points) whereas HA subjects averaged $91 \%$ correct (SD: 15.90 percentage-points) on this test. An independent-samples $t$ test showed a significant difference between the scores of the two groups ( $p<$ 0.001).

\section{Correlations}

Nonparametric Spearman $\rho$ calculations were performed for the CI and HA subject groups to examine correlations between the mean scores on the melody test, and the mean scores on the pitch and the rhythm tests. For the CI subjects, there was a significant moderate correlation between their melody test score and the mean score across all three pitch subtests ( $\rho=0.679, p=0.003$ ) (Fig. 2). For the HA group, there were no significant correlations between the melody and pitch test mean scores. There were no significant correlations between the rhythm and melody test scores for any group. There were also no significant correlations between the music test battery scores and the subject factors of age, music experience, device experience, or speech perception scores, for either group.

\section{Learning Effect}

As it was observed that for both subject groups, scores were generally higher for test block 2 than test block 1, paired $t$ tests were conducted to compare the two sets of scores for the CI and HA groups separately. This was to assess for any learning effect. For the CI subject group, there was no significant difference between the scores obtained for the

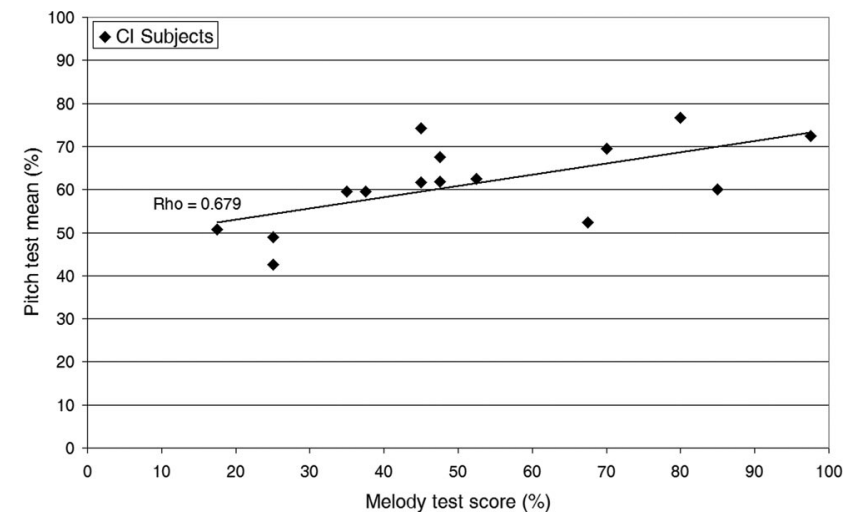

Fig. 2. Correlation between the scores on the melody test and mean of the three pitch subtests for the $\mathrm{Cl}$ subject group. two test blocks, for any of the tests. For the HA group, there was no significant difference between the two administrations of the rhythm, melody, or the ensemble identification tests/subtests. However, the scores from test block 2 were significantly higher than those from test block 1 for the pitch test $(p<$ 0.001), the single-instrument identification subtest $(p=0.046)$, and the identification of instruments with background accompaniment subtest ( $p=$ 0.006). Post hoc paired $t$ tests for the pitch test showed significant differences between the test blocks for all three interval sizes (one-octave: $p=$ 0.034; half-octave: $p=0.003$; quarter-octave: $p=$ 0.004). In all three cases, test block 2 scores were higher than test block 1 scores.

\section{Discussion}

The results of this research partially verified the initial hypothesis that HA users who met the CI candidacy criteria would perform better than CI recipients on pitch discrimination, instrument identification, and melody recognition tests, but similarly on rhythm perception tests. Consistent with previous research, the CI subjects in this study found tests involving pitch, instrument, or melody perception significantly more difficult than those involving just rhythm perception (Dorman, et al., 1991; Fujita \& Ito, 1999; Gfeller \& Lansing, 1991, 1992; Gfeller, et al., 1997, 1998, 2002a,c; Leal, et al., 2003; McDermott, 2004; Schulz \& Kerber, 1994). As hypothesized, there was no significant difference between the CI and HA subjects on the rhythm test. The CI group scored significantly lower on the pitch and melody tests ( $p<0.001$ for both comparisons); however, the two groups scored equivalently on the instrument recognition tests. The melody recognition results showed a significant disparity between the results for the HA and CI groups, with a wide range of individual scores, consistent with previous studies (Gfeller, et al., 2002a, 2005; Kong, et al., 2005). Scores on the music test battery were not significantly correlated with any subject variable such as age, music experience, device experience, or speech perception scores, for either subject group.

The overall results of the pitch test indicate that the CI does not provide wearers with sufficiently reliable pitch cues for them to consistently discriminate between two musical notes. The CI subject group obtained lower pitch-ranking scores than the HA group for all interval sizes tested, only scoring at chance levels for the quarter-octave stimuli (frequency ratio $\sim 19 \%$ ). That is, as a group, the CI subjects were unable to discriminate between notes three semitones apart. This difficulty in accurately perceiving pitch could have contributed to the sig- 
nificantly lower melody recognition results of the CI subject group in comparison with the HA subject group; melody recognition would require at least some degree of accurate pitch perception.

Perceiving the pitch of a complex sound primarily involves the listener having to extract fundamental frequency (F0) information from the complex acoustic signal. For a CI user to extract this information, one of two different mechanisms can be used-(1) resolving the individual frequency components present in the signal, and/or (2) extracting the temporal pitch information from the signal. Hence, the difficulty experienced by CI users on pitch-based tasks may arise for a host of reasons, as discussed below.

CIs were designed on the premise that activating different electrode sites should result in different pitch percepts in accordance with the tonotopic structure of the cochlea. However, current filterbanks of the CI22 and CI24 implants only allow for a maximum of 22 overlapping filterbands with fixed center frequencies. The widths of these filters vary depending on the number of filters being used and the center frequency of each filter. A wide filter may preclude the lower harmonics of complex sounds from being fully resolved, making it difficult for the listener to determine the harmonic frequencies, and make reliable pitch judgments. For example, for the sung vowel with the lowest F0 used in this study (98 $\mathrm{Hz}$ ), there would have been no harmonics resolved by the Nucleus CI's filterbank, assuming that the default filter-assignment table was implemented in the device. Even if the individual harmonic components were resolved, falling into separate filters, the CI user may only be able to determine which filter the component passed to, as the corresponding electrode would be activated. However, they would be unable to use place cues to determine the precise position of the signal within that filter's bandwidth and accordingly, the exact frequency of the resolved component. Further, if the resolved components were in adjacent filters that subsequently activated two or more adjacent electrodes, the CI user may not be able to resolve the places of stimulation to accurately determine pitch information.

This inaccuracy in pitch perception resulting from poor frequency resolution might be further confounded by a mismatch between the frequency of the CI's filter and the corresponding characteristic frequency in the cochlea. With typical electrode insertion depths extending to the first 1.5 turns of the cochlea only, filterbands assigned to active electrodes tend to be lower in frequency than the characteristic frequency normally associated with that stimulation site.

Instead of resolving the signal's frequency components, CI users may also use temporal cues to determine the pitch of a complex sound. For the
Nucleus implants worn by subjects in this study, temporal cues are available via amplitude modulations present at the filterbank's output, at a rate corresponding to the input signal's F0; these amplitude modulations can provide a pitch percept (McKay \& McDermott, 1996; McKay, et al., 1994, 1995; Pijl, $1995,1997)$. The availability and clarity of these modulations are contingent on having a filter wide enough to encompass more than one harmonic, an adequately high stimulation rate to permit accurate sampling, a sufficiently deep modulation depth, and the filter's output not being smoothed by the processing strategy. The salience of these amplitude-modulation cues is further dependent on the alignment of the phase of these modulations across electrode positions. If phase misalignments are present at the filterbank's output, the resulting overall modulation pattern may no longer reflect the F0 if the modulations are perceptually combined across electrode positions. Research by McKay \& McDermott indicates that CI users may integrate temporal modulations across closely spaced active electrodes, resulting in inaccurate temporal cues. It also seems that CI users are only able to extract reliable pitch cues from these amplitude modulations at frequencies up to around $300 \mathrm{~Hz}$, implying that the majority of CI users could have difficulty in obtaining reliable pitch cues from temporal variations in stimuli with a F0 above approximately middle-C (McKay, 2004; McKay, et al., 1994, 1995; Pijl, 1997; Zeng, 2002).

The accuracy of CI users' responses in pitch-ranking tasks may also depend on which cues the subject uses in making their perceptual judgments. Researchers have suggested that place- and temporal-based cues provide two perceptually independent sensations to the listener (McDermott, 2004; McDermott \& McKay, 1997; Moore \& Carlyon, 2005; Pijl \& Schwarz, 1995). In making perceptual decisions, subjects may apply different weightings to these cues depending on their salience and availability during each task. Should the cues provide conflicting or incorrect information, the implant user's ability to make accurate pitch decisions could additionally be affected. This will be further discussed below.

Along with aforementioned issues, a range of environmental, physiological, and pathological considerations could also affect an individual implantee's ability to perceive pitch. Examples of these variables include their memory for melodic pitches, music knowledge or training; the location, number, and density of surviving neurons in the cochlea; the electrode's placement or insertion depth; the impedance surrounding the electrodes; pathological processes; central processing factors; and the stimulation mode used or electrical current path within the cochlea (McDermott, 2004; McKay, 2004, 2005; McKay \& 
McDermott, 1993; Pijl, 1995). All these factors contribute to the large variability in the pitch perception skills of individual implantees as reported in previous research (Fujita \& Ito, 1999; Gfeller, et al., 1997, 2002a; McDermott, 2004; Schulz \& Kerber, 1994). This variability was also evident in the current study. For example, for the one-octave subtest, the CI group's mean scores ranged from $24 \%$ to $91 \%$ correct with a standard deviation of 17 percentagepoints. When each subject's percent-correct scores were averaged for both test blocks across the four vowels, 9 of the 15 subjects scored significantly different from the chance score of $50 \%$ for this subtest. In contrast, the HA group's mean scores for this same subtest ranged from $76 \%$ to $98 \%$ correct, with a standard deviation of 10 percentage-points. All the HA subjects' four-vowel means were significantly different from the chance score of $50 \%$.

Despite being higher than the scores obtained from the CI group, the pitch results from the HA subject group were not as good as what one may expect from NH listeners. Schulz \& Kerber (1994) and Gfeller et al. (2002a) found that the majority of the $\mathrm{NH}$ controls in their studies could reliably rank pitches one semitone apart. In the current study, the group of $\mathrm{NH}$ adults who verified the music tests scored greater than 95\% correct when ranking pitches three semitones apart. The HA group only scored $75 \%$ correct. A number of researchers have reported reduced frequency selectivity arising from increased auditory filter bandwidths in listeners with cochlear hearing losses (Arehart, 1994; Moore, 1995, 1996; Moore \& Peters, 1992; Summers \& Leek, 1994). Moore (1996) reported that hearing thresholds worse than approximately 40 to $50 \mathrm{~dB}$ HL result in auditory filters with bandwidths approximately two times wider than those for NH. The reduced frequency selectivity due to the wider filter bandwidths may have a deleterious effect on pitch-based tests as the listener would be less able to resolve the lower-order harmonics, affecting their perception of the F0. This finding suggests the possibility that some of the disparity reported between the pitch perception results of $\mathrm{CI}$ users and $\mathrm{NH}$ subjects is attributable to physiological differences arising from having a significant hearing loss.

The issues related to temporal-pitch perception may also account for the greater accuracy demonstrated by the CI subjects for male-sung stimuli than those sung by a female voice. In the pitch test, the CI group was significantly better at ranking the vowels sung by the male vocalist than the female vocalist. With temporal cues only being available for frequencies lower than approximately 200 to 300 $\mathrm{Hz}$, the lower F0s associated with male voices may have enabled the CI subjects to use temporal mod- ulation cues in making pitch judgments. The femalesung vowels in this study would have had higher F0, though, and two possibilities arise as to why pitch discrimination was less accurate for these stimuli. First, as previously mentioned, both temporal and place changes may have been perceived by the implant user, but the two types of change provided conflicting information, making ranking unreliable and inconsistent. For example, it is possible that, for an ascending pair of notes, the electrode stimulation could shift apically as a result of downward changes in formant frequencies; in general, changes in formant frequencies need not be related monotonically to changes in the F0 of sung vowels (Maurer \& Landis, 1995). This would be contrary to the increased amplitude-modulation rate, corresponding to the increase in the F0. In this case, a pitch reversal would result for a subject attending to place. More generally, there may be no changes in stimulation place related systematically to changes in the F0. Alternatively, it may have been possible that while the F0 exceeded the subject's upper limit for using temporal-pitch cues, the difference between the F0 of the pitches in the stimulus pair was not sufficiently large to result in a change in the place of stimulation to provide a pitch cue. With place cues at the output of the processor being related to the peaks in the spectral envelope, it is possible that there were no place-cue changes related to the shift in the F0.

In contrast to the CI users, the HA subject group scored significantly better for the female-sung vowels than the male-sung vowels $(p=0.001)$. The lower harmonics of sounds with higher F0s are more likely to be fully resolved, falling into separate auditory filters. Thus the higher F0s for the female-sung vowels could have resulted in a larger number of resolvable harmonics to provide place-pitch cues. Research suggests that for a listener with $\mathrm{NH}$, the resolved harmonics are important for extracting the pitch percept (Moore \& Carlyon, 2005).

For the CI subjects, the ability to rank pitches was associated with the ability to recognize melodies, with significant moderate correlations being obtained between the scores on the melody test and the mean score across the three pitch subtests. This is consistent with the results of Looi et al. (2004), suggesting that implantees use more than just rhythm cues to identify familiar melodies. In the current study, there was no significant correlation between the rhythm and melody test scores for either group, probably because of the ceiling effect for the rhythm test. For the HA group, the lack of a significant correlation between the melody and pitch test scores was possibly also attributable to a ceiling effect for the melody test; 19 of the 30 separate 
melody test scores (15 subjects, 2 runs each) were either $100 \%$ or $95 \%$, corresponding to a maximum of one error on the test.

Accurate timbre perception requires the perception of both the signal's temporal envelope, and the energy spectrum of its harmonic components. Modifying features of the temporal envelope or changing the frequencies and/or amplitude of the harmonic components could alter the timbre perceived (Handel, 1989; Kohlrausch \& Houtsma, 1989). For the instrument identification subtests, although it was observed that the HA group scored higher than the CI group for all three subtests, these differences were not statistically significant. The lack of difference between the two groups in the instrument recognition test is a somewhat surprising finding, considering the contrasting modes of auditory stimulation involved-i.e., acoustic hearing for the HA users versus electric hearing for the CI subjects. The underlying reasons for the difficulty experienced in perceiving the signal's envelope would differ for acoustic and electric hearing; however, the resulting effect on the tasks of instrument identification was similar. Seemingly, the better pitch perception skills of the HA subject group did not translate into improved timbre perception. Given that the $\mathrm{NH}$ adults who verified the subtests averaged above $95 \%$, the comparatively poorer identification results for the hearing-impaired subject groups suggests that neither device sufficiently transmits the broad spectral envelope and/or temporal envelope information from the input signal to enable accurate timbral perception. This may have arisen from a range of factors.

For an NH individual, such spectral selectivity derives from the different frequency components of the acoustic stimulus being separated into different auditory filters, with each frequency component resulting in activity at discrete sites along the basilar membrane. However, for CI users, the degree of discreteness of electrode stimulation sites, and thus the spectral selectivity, is not nearly as precise as for NH. Henry et al. (2005) found that spectral peak resolution for rippled-noise stimuli was best for the subjects with $\mathrm{NH}$, intermediate for those with hearing impairments, and worst for those using an implant. The perceptual smearing experienced by CI users may also be a by-product of the stimulation itself, such as the presence of channel interactions, or it may arise from the use of nonlinear amplitude mapping functions in converting acoustic signals to appropriate levels for electrical stimulation (Laback, et al., 2004; McDermott, 2004; McKay, 2004). Such spectral smearing, in combination with the coarse spectral analysis of the input signal undertaken by current pulsatile speech-processing strategies such as ACE and SPEAK, may in part account for the difficulty experienced by CI users in tasks related to timbre perception, including the identification of musical instruments. For HA users, perceptual smearing may occur as a consequence of auditory filter anomalies associated with cochlear hearing loss, poor neural survival patterns, and poor frequency selectivity. This may result in diminishing the spectral clarity of the stimuli for the subject (Arehart, 1994; Moore, 1995; Summers \& Leek, 1994).

The high levels of intersubject variability on the instrument perception tasks documented by previous researchers (Gfeller, et al., 1998, 2002c; McDermott \& Looi, 2004) was also observed in the current study for both the CI and HA subjects. In the single-instrument identification subtest, the CI subjects averaged $61 \%$ correct with a standard deviation of 11 percentage-points, whereas the HA subjects averaged $69 \%$ correct with a standard deviation of 12 percentage-points. Comparisons of the absolute identification scores obtained in the current study to previous research results are confounded by highly varying methodologies and test requirements between studies. However, despite these methodological differences, the inequality in performance between the $\mathrm{CI}$ and $\mathrm{NH}$ subjects is consistently evident across existing studies, with the current study also suggesting that HA subjects with significant levels of hearing impairment may similarly experience difficulty on instrument perception tasks when compared with listeners with $\mathrm{NH}$.

This study also extended the investigation of timbre perception beyond the single-instrument identification tasks used in most previous studies. Results from the "instrument with background accompaniment" and "music ensemble" subtests reflected the more complex nature of these excerpts. Statistical analyses showed significant differences between the identification scores for the first subtest using single-instrument stimuli and both of the other subtests using multi-instrumental stimuli (CI and HA subject groups-subtests 1 and 2: $p<0.001$; subtests 1 and 3: $p<0.001$ ). The additional instruments present in the second and third subtests added to the complexity of the sound, which seemed to negatively affect a hearing-impaired subjects' perception of the stimuli, irrespective of whether they used a CI or HA.

The poor pitch and timbre perception results for the CI subjects in this study are in-keeping with existing literature findings. Accordingly, researchers have been designing and evaluating a number of different sound-processing strategies or techniques to improve the perception of pitch and timbre through the CI. Some of these have focused on providing more temporal information; for example, by providing more fine-structure information, using very high stimulation rates, enhancing the F0 modulation depths, rep- 
resenting the $\mathrm{F} 0$ by changing the stimulation rate, eliminating the phase shifts that occur when information is combined across electrode positions, or using higher carrier, and/or sampling rates (Geurts \& Wouters, 2004; Kessler, 1999; Laneau, et al., 2006; Loizou, et al., 2003; McDermott, 2004; Nie, et al., 2005; Vandali, et al., 2005; Wilson, 2004; Wilson, et al., 2003; Zeng, 2004). Others have aimed to improve spectral resolution, such as by modifying the filterbank of the speech processor (Kasturi \& Loizou, Reference Note 1), or using "virtual channels" to give finer frequency resolution (Donaldson, et al., 2005; Kasturi \& Loizou, Reference Note 2; Poroy \& Loizou, Reference Note 3; Zeng, 2004). Reported results have shown mixed success. As yet, no particular approach has been consistently better than others nor has any approach consistently improved pitch perception by CI users.

In view of the finding that HAs may provide more reliable F0 information than CIs, which enhances pitch perception, whereas the CI potentially provides additional high-frequency information, the combination of the two devices may warrant further investigation. The use of electroacoustic stimulation has been recommended as a potentially effective way of improving F0 perception for certain CI users who have sufficient levels of residual hearing (Gantz \& Turner, 2004; Kong, et al., 2005). The use of acoustic hearing enables some of the lower-frequency finestructure cues to be perceived, thereby increasing the potential for $\mathrm{F} 0$ information to be extracted.

It is also worth briefly mentioning the learning effect observed between the two test runs of the music test battery in this study, with higher test scores on the second test block being recorded, for some subtests. Although the difference was only statistically significant for the HA group's results for the pitch test, and the first two instrument identification subtests, the pattern was observed for both subject groups across all of the tests and subtest scores, except for the rhythm test. The learning effect in this study was task-specific, and related to task-familiarity; this study did not include any form of music rehabilitation or a structured training program. It would be interesting, though, to investigate whether specialized training could benefit music perception, with previously conducted research suggesting that a music training program could benefit some subjects who may want to improve their music perception skills (Gfeller, 2001; Gfeller, et al., 2000, 2002b).

\section{Conclusions}

This study compared various aspects of music perception between CI users and HA users with similar levels of hearing loss. The results obtained partially supported the initial hypothesis that HA users who met the CI candidacy criteria would perform better than CI recipients on pitch discrimination, instrument identification, and melody recognition tests, but similarly on rhythm perception tests. There was no significant difference between the CI and HA subjects on the rhythm test. The CI group scored significantly lower on the pitch and melody tests $(p<0.001)$, but the two groups scored equivalently on the instrument recognition tests. Previous research has shown that $\mathrm{NH}$ listeners are significantly better than CI users at pitch-based tasks. This study found a significant disparity between the pitch perception skills of CI users and HA users with similar levels of hearing loss. It may be inferred, then, that replacing an $\mathrm{HA}(\mathrm{s})$ with a CI has an adverse affect on some aspects of music perception, and that wearing an HA simultaneously with the CI is likely to improve music perception over wearing a CI alone. The pattern of results was also different for the two groups; the CI group scored higher for the male-sung than female-sung vowels, in contrast to the HA group who scored higher for the female-sung vowels.

Based on these poor pitch perception results, it follows that the melody perception scores for CI subjects may also be affected as accurate perception of western music requires the listener to discriminate between intervals one semitone apart. This was shown to be the case for the CI subject group, whose scores on the melody test were significantly poorer than those obtained by the HA subject group. The CI subject group were only able to recognize just over half (52\%) of the melodies presented, even though these melodies included both pitch and rhythm cues.

There was no significant difference between the $\mathrm{CI}$ and HA groups in the instrument recognition test, despite the contrasting modes of auditory stimulation involved. For both groups, instrument recognition deteriorated as stimuli complexity increased; performance was significantly better for single-instrument stimuli than multiple-performer instrumentations.

Although the HA users in this study who had significant levels of hearing loss performed better than the CI users on some tasks, their results suggest that they may not achieve optimal music perception either. Although the pitch perception scores for subjects using an HA were better than those obtained by the CI users, the performance of the former was still significantly poorer than that achieved by $\mathrm{NH}$ listeners. It is therefore possible that a portion of the disparity reported in existing research comparing $\mathrm{CI}$ users and $\mathrm{NH}$ subjects may be related to physiological differences that arise from having a significant hearing loss. 
In summary, the results of the experiments indicate that for these music tests, HA users with similar levels of hearing loss perform at least equal to, and for the tasks of pitch perception, better than, CI users. This should be considered when counseling prospective CI recipients with regard to postsurgery outcomes and expectations. However, despite the differences between scores obtained by the CI and HA subject groups, both of these subject groups were largely unable to perceive music accurately, regardless of the device they used. These findings support the need for ongoing research into the music perception of people with hearing impairments, be they CI recipients and/or HA users.

\section{ACKnowledgments}

The authors acknowledge Karen Pedley, Greg Angus and the staff at Queensland Hearing, the Cochlear Implant Clinic at the Royal Victorian Eye and Ear Hospital, and Australian Hearing for assistance with recruiting subjects; Dr. Ross Darnell from the School of Health and Rehabilitation Sciences at the University of Queensland, and Associate Professor Ian Gordon from the Statistical Consulting Centre at the University of Melbourne for assistance with statistical analysis; Dr. Waikong Lai for the software used for testing subjects; as well as colleagues Catherine Sucher and Andrea Simpson for their invaluable assistance. Finally, the authors thank all the subjects for their time and contribution to this study.

This research was supported by the National Health and Medical Research Council of Australia, the Cooperative Research Centre for Cochlear Implant and Hearing Aid Innovation, and the Garnett Passe and Rodney Williams Memorial Foundation.

Address for correspondence: Valerie Looi, Department of Communication Disorders, The University of Canterbury, Private Bag 4800, Christchurch 8020, New Zealand. E-mail: valerie.looi@ canterbury.ac.nz.

Received November 20, 2006; accepted October 31, 2007.

\section{REFERENCES}

Arehart, K. H. (1994). Effects of harmonic content on complextone fundamental-frequency discrimination in hearing-impaired listeners. J Acoust Soc Am, 95, 3574-3585.

Darrow, A. (1979). The beat reproduction response of subjects with normal and impaired hearing: an empirical comparison. J Music Ther, 16, 91-98.

Darrow, A. (1984). A comparison of rhythmic responsiveness in normal and hearing impaired children and an investigation of the relationship of rhythmic responsiveness to the suprasegmental aspects of speech perception. J Music Ther, 21, 48-66.

Donaldson, G. S., Kreft, H. A., \& Litvak, L. (2005). Place-pitch discrimination of single-versus dual-electrode stimuli by cochlear implant users. J Acoust Soc Am, 118, 623-626.

Dorman, M. F., Basham, K., McCandless, G., et al. (1991). Speech understanding and music appreciation with the Ineraid cochlear implant. Hear J, 44, 34-37.

Fujita, S., \& Ito, J. (1999). Ability of nucleus cochlear implantees to recognize music. Ann Otol Rhinol Laryngol, 108, 634-640.

Gantz, B. J., \& Turner, C. (2004). Combining acoustic and electrical speech processing: Iowa/Nucleus hybrid implant. Acta Otolaryngol, 124, 344-347.
Geurts, L., \& Wouters, J. (2004). Better place-coding of the fundamental frequency in cochlear implants. J Acoust Soc Am, $115,844-852$.

Gfeller, K. (2001). Aural rehabilitation of music listening for adult cochlear implant recipients: addressing learner characteristics. Mus Ther Perspect, 19, 88-95.

Gfeller, K., Knutson, J. F., Woodworth, G., et al. (1998). Timbral recognition and appraisal by adult cochlear implant users and normal-hearing adults. J Am Acad Audiol, 9, 1-19.

Gfeller, K., \& Lansing, C. (1992). Musical perception of cochlear implant users as measured by the Primary Measures of Music Audiation: an item analysis. J Music Ther, 29, 18-39.

Gfeller, K., \& Lansing, C. R. (1991). Melodic, rhythmic, and timbral perception of adult cochlear implant users. J Speech Hear Res, 34, 916-920.

Gfeller, K., Olszewski, C., Rychener, M., et al. (2005). Recognition of "real-world" musical excerpts by cochlear implant recipients and normal-hearing adults. Ear Hear, 26, 237-250.

Gfeller, K., Turner, C., Mehr, M., et al. (2002a). Recognition of familiar melodies by adult cochlear implant recipients and normal-hearing adults. Cochlear Implants Int, 3, 29-53.

Gfeller, K., Witt, S., Adamek, M., et al. (2002b). Effects of training on timbre recognition and appraisal by postlingually deafened cochlear implant recipients. J Am Acad Audiol, 13, 132-145.

Gfeller, K., Witt, S., Stordahl, J., et al. (2000). The effects of training on melody recognition and appraisal by adult cochlear implant recipients. J Acad Rehabil Audiol, 33, 115-138.

Gfeller, K., Witt, S., Woodworth, G., et al. (2002c). Effects of frequency, instrumental family, and cochlear implant type on timbre recognition and appraisal. Ann Otol Rhinol Laryngol, 111, 349-356.

Gfeller, K., Woodworth, G., Robin, D. A., et al. (1997). Perception of rhythmic and sequential pitch patterns by normally hearing adults and adult cochlear implant users. Ear Hear, 18, 252260.

Gordon, E. E. (1986). Manual for the Primary Measures of Music Audiation and the Intermediate Measures of Music Audiation. Chicago: GIA Publications.

Handel, S. (1989). Listening: an introduction to the perception of auditory events. Cambridge: MIT Press.

Henry, B. A., Turner, C. W., \& Behrens, A. (2005). Spectral peak resolution and speech recognition in quiet: normal hearing, hearing impaired, and cochlear implant listeners. J Acoust Soc Am, 118, 1111-1121.

Kessler, D. K. (1999). The CLARION multi-strategy cochlear implant. Ann Otol Rhinol Laryngol, 108, 8-16.

Klajman, S., Koldej, E., \& Kowalska, A. (1982). Investigation of musical abilities in hearing-impaired and normal hearing children. Folia Phoniatr (Basel), 34, 229-233.

Kohlrausch, A., \& Houtsma, A. J. M. (1989). Description of complex sounds. In S. Nielzen, \& O. Olsson (Eds.), Structure and perception of electroacoustic sound and music (pp. 141159). Amsterdam: Excerpta Medica.

Kong, Y. Y., Stickney, G. S., \& Zeng, F. G. (2005). Speech and melody recognition in binaurally combined acoustic and electric hearing. J Acoust Soc Am, 117(3 Pt 1), 1351-1361.

Korduba, O. M. (1975). Duplicated rhythm patterns between deaf and normal hearing children. J Music Ther, 12, 136-146.

Laback, B., Deutsch, W. A., \& Baumgartner, W. D. (2004). Coding of vowellike signals in cochlear implant listeners. J Acoust Soc Am, 116, 1208-1223.

Laneau, J., Wouters, J., \& Moonen, M. (2006). Improved music perception with explicit pitch coding in cochlear implants. Audiol Neurootol, 11, 38-52.

Leal, M. C., Shin, Y. J., Laborde, M. L., et al. (2003). Music perception in adult cochlear implant recipients. Acta Otolaryngol, 123, 826-835. 
Loizou, P. C., Stickney, G., Mishra, L., et al. (2003). Comparison of speech processing strategies used in the Clarion implant processor. Ear Hear, 24, 12-19.

Looi, V., McDermott, H. J., McKay, C. M., et al. (2004). Pitch discrimination and melody recognition by cochlear implant users. In R. Miyamoto (Ed.), Cochlear implants: proceedings of the VIII international cochlear implant conference. International Congress Series (Vol. 1273C, pp. 197-200). Amsterdam: Elsevier.

Looi, V., Sucher, C., \& McDermott, H. J. (2003). Melodies familiar to the Australian population across a range of hearing abilities. Aust New Zeal J Audiol, 25, 75-83.

Maurer, D., \& Landis, T. (1995). F0-dependence, number alteration, and non-systematic behaviour of the formants in German vowels. Int J Neurosci, 83, 25-44.

McDermott, H. J. (2004). Music perception with cochlear implants: a review. Trends Amplif, 8, 49-82.

McDermott, H. J., \& Looi, V. (2004). Perception of complex signals, including musical sounds, with cochlear implants. In R. Miyamoto (Ed.), Cochlear implants: proceedings of the VIII international cochlear implant conference. International Congress Series (Vol. 1273C, pp. 201-204). Amsterdam: Elsevier.

McDermott, H. J., \& McKay, C. M. (1997). Musical pitch perception with electrical stimulation of the cochlea. J Acoust Soc Am, 101, 1622-1631.

McKay, C. M. (2004). Psychophysics and electrical stimulation. In F. G. Zeng, A. N. Popper, \& R. R. Fay (Eds.), Cochlear implants: auditory prostheses and electric hearing (Vol. 20, pp. 286-333). New York: Springer.

McKay, C. M. (2005). Spectral processing in cochlear implants. In M. S. Malmierca \& D. R. F. Irvine (Eds.), Auditory spectral processing (Vol. 70, pp. 474-509). London: Academic Press.

McKay, C. M., \& McDermott, H. J. (1993). Perceptual performance of subjects with cochlear implants using the Spectral Maxima Sound Processor (SMSP) and the Mini Speech Processor (MSP). Ear Hear, 14, 350-367.

McKay, C. M., \& McDermott, H. J. (1996). The perception of temporal patterns for electrical stimulation presented at one or two intracochlear sites. J Acoust Soc Am, 100 (2 Pt 1), 10811092.

McKay, C. M., McDermott, H. J., \& Clark, G. M. (1994). Pitch percepts associated with amplitude-modulated current pulse trains in cochlear implantees. J Acoust Soc Am, 96 (5 Pt 1), 2664-2673.

McKay, C. M., McDermott, H. J., \& Clark, G. M. (1995). Pitch matching of amplitude-modulated current pulse trains by cochlear implantees: the effect of modulation depth. J Acoust Soc Am, 97, 1777-1785.

Moore, B. C. J. (1995). Perceptual consequences of cochlear damage. Oxford: Oxford University Press.

Moore, B. C. J. (1996). Perceptual consequences of cochlear hearing loss and their implications for the design of hearing aids. Ear Hear, 17, 133-161.

Moore, B. C. J., \& Carlyon, R. P. (2005). Perception of pitch by people with cochlear hearing loss and by cochlear implant users. In C. J. Plack, A. J. Oxenham, R. R. Fay, \& A. N. Popper
(Eds.), Pitch: neural coding and perception (Vol. 24, pp. 234277). New York: Springer-Verlag.

Moore, B. C. J., \& Peters, R. W. (1992). Pitch discrimination and phase sensitivity in young and elderly subjects and its relationship to frequency selectivity. J Acoust Soc Am, 91, 2881-2893.

Nie, K., Stickney, G., \& Zeng, F. G. (2005). Encoding frequency modulation to improve cochlear implant performance in noise. IEEE Trans Biomed Eng, 52, 64-73.

Pijl, S. (1995). Musical pitch perception with pulsatile stimulation of single electrodes in patients implanted with the Nucleus cochlear implant. Ann Otol Rhinol Laryngol Suppl, 166, 224227.

Pijl, S. (1997). Labeling of musical interval size by cochlear implant patients and normally hearing subjects. Ear Hear, 18, 364-372.

Pijl, S., \& Schwarz, D. W. (1995). Melody recognition and musical interval perception by deaf subjects stimulated with electrical pulse trains through single cochlear implant electrodes. $J$ Acoust Soc Am, 98 (2 Pt 1), 886-895.

Schulz, E., \& Kerber, M. (1994). Music perception with the MED-EL implants. In I. J. Hochmair-Desoyer, \& E. S. Hochmair (Eds.), Advances in cochlear implants (pp. 326-332). Vienna: Datenkonvertierung, Reproduktion und Druck.

Summers, V., \& Leek, M. R. (1994). The internal representation of spectral contrast in hearing-impaired listeners. J Acoust Soc Am, 95, 3518-3525.

Vandali, A. E., Sucher, C., Tsang, D. J., et al. (2005). Pitch ranking ability of cochlear implant recipients: a comparison of sound-processing strategies. J Acoust Soc Am, 117, 3126-3138.

Wilson, B. S. (2004). Engineering design of cochlear implants. In F. G. Zeng, A. N. Popper, \& R. R. Fay (Eds.), Cochlear implants: auditory prostheses and electric hearing (Vol. 20, pp. 14-52). New York: Springer.

Wilson, B. S., Lawson, D. T., Muller, J. M., et al. (2003). Cochlear implants: some likely next steps. Annu Rev Biomed Eng, 5, 207-249.

Zeng, F. G. (2002). Temporal pitch in electric hearing. Hear Res, 174, 101-106.

Zeng, F. G. (2004). Trends in cochlear implants. Trends Amplif, 8, $1-34$.

\section{REFERENCE Notes}

1 Kasturi, K., \& Loizou, P. C. (2005). Effect of filter spacing and correct tonotopic representation on melody recognition: implications for cochlear implants. Paper presented at the conference for the Association for Research in Otolaryngology, New Orleans, USA.

2 Kasturi, K., \& Loizou, P. C. (2005). Optimizing virtual channel selection. Paper presented at the 2005 conference on Implantable Auditory Prostheses, July 30-Aug 4 2005, Asilomar, Monterey, California, USA.

3 Poroy, O., \& Loizou, P. C. (2001). Pitch perception of virtual channels. Paper presented at the 2001 conference on Implantable Auditory Prostheses, Asilomar, Monterey, California, USA. 